\title{
Signalling antibodies for the treatment of neoplastic disease
}

\author{
M.J. Glennie \\ Tenovus Research Laboratory, The Cancer Sciences \\ Division, Southampton University School of Medicine, \\ Southampton SO16 6YD, UK \\ E-mail:mjg@soton.ac.uk
}

It is 25 years since Kohler and Milstein first described making monoclonal antibodies using somatic cell fusion. While this technology has had a critical influence on the diagnosis and classification of cancer, it is only in the last 5 years that we have started to see its impact on cancer treatment. Several clinical trials now show that $\mathrm{mAb}$ can deliver therapeutic activity which benefits patients without long-term toxicity, and this has resulted in two reagents, Rituximab and Herceptin, being FDA approved for clinical application.

This major turnaround appears to have occurred as a result of two factors: first, the realisation of the critical importance of selecting the appropriate target on tumour cells, since it is clear that most mAb are not therapeutic; and second, the genetic engineering that has allowed the production of human/rodent chimeric $\mathrm{mAb}$. These engineered reagents carry human constant regions which are less immunogenic than rodent $\mathrm{mAb}$, recruit human effectors more efficiently and survive in the circulation with an extended half-life.

Developing further improvements in anti-cancer $\mathrm{mAb}$ demands that we know more about the mechanisms by which they operate in vivo. Conventional theories argue that a therapeutic antibody, once bound to a cell surface, operates by recruiting cytotoxic effector systems, such as lymphocytes, macrophages and complement, in a way that is similar to the killing seen following infection by a microbial pathogen. However, a growing body of evidence now shows that mAb have other roles, including the ability to crosslink surface receptors and thereby generate transmembrane signals in the tumour cells. Providing the mAb has the correct specificity, such crosslinking can deliver growth con- trol and cell death signals to tumour. There is some data to suggest that anti-CD20 (Rituximab) may utilise this mechanism against lymphoma. Taking this idea even further, an exciting new group of mAb has recently been defined that not only crosslink target molecules but actually stimulate active anti-tumour immunity in the host. Included in this group are anti-CTLA-4, antiCD40, anti-BAT and anti-CD137. By crosslinking or blocking key cell surface molecules, these mAb are able to induce powerful T-cell responses that destroy tumours and leave the host immune to further challenge. In most cases these $\mathrm{mAb}$ bind to targets on CD4, CD8 or APC, and appear to mimic the action of the natural ligand of the target molecule. For example, anti-CD40 mAb, which is one of the most active of this group, probably mimics the action of the CD40L on helper T cells, and, by activating CD40+ APC, promotes the efficiency with which tumour antigens are processed and presented to CD8 cytotoxic T cells. In contrast, anti-CTLA4 works by blocking the inhibitory effects of CTLA4-B7 interaction and thereby allows T-cell costimulation via CD28 to predominate in the tumour response. Regardless of the initiating mechanism, treatment with such $\mathrm{mAb}$ results in rapid and in some cases massive expansion of CD8 cytotoxic T cells that are capable of destroying the tumours, and leaves animals partially or completely immune against tumour re-challenge. The next step will be to discover whether active immunity can be achieved in patients, where tumour loads are usually greater and have also evolved to escape immune detection, and where tumours may be less immunogenic.

\section{References}

[1] M.S. Cragg, R.R. French and M.J. Glennie, Signalling antibodies in cancer therapy, Current Opinions Immunology 5 (1999), 541-547.

[2] M.J. Glennie and P.W.M. Johnson, Clinical trials of antibody therapy, Immunology Today 21(8) (2000), 403-410. 


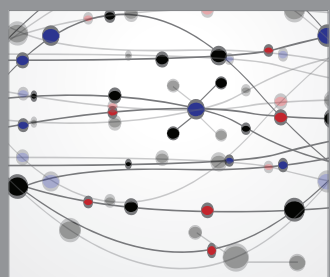

The Scientific World Journal
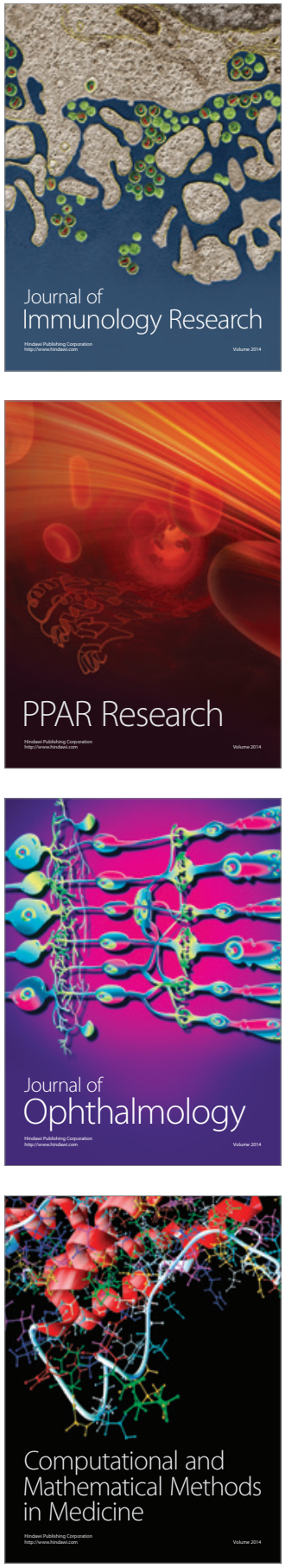

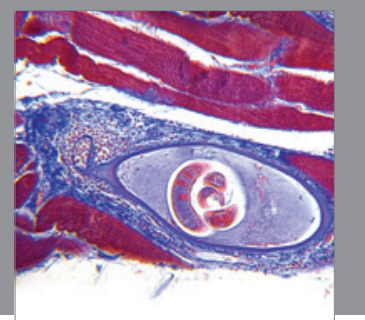

Gastroenterology

Research and Practice
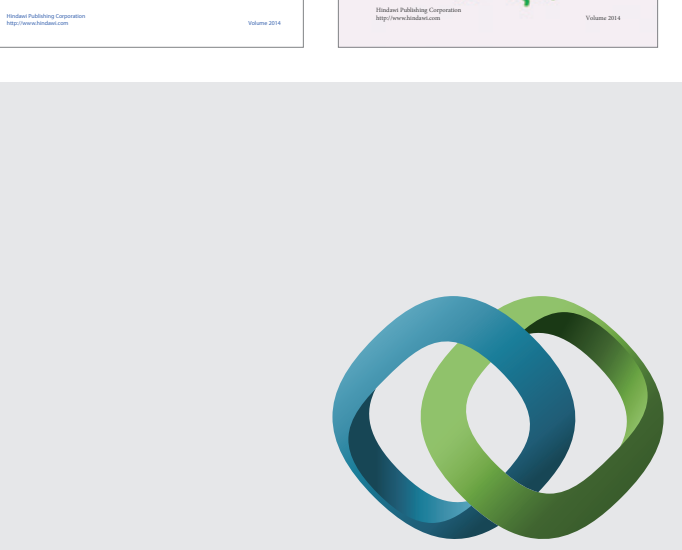

\section{Hindawi}

Submit your manuscripts at

http://www.hindawi.com
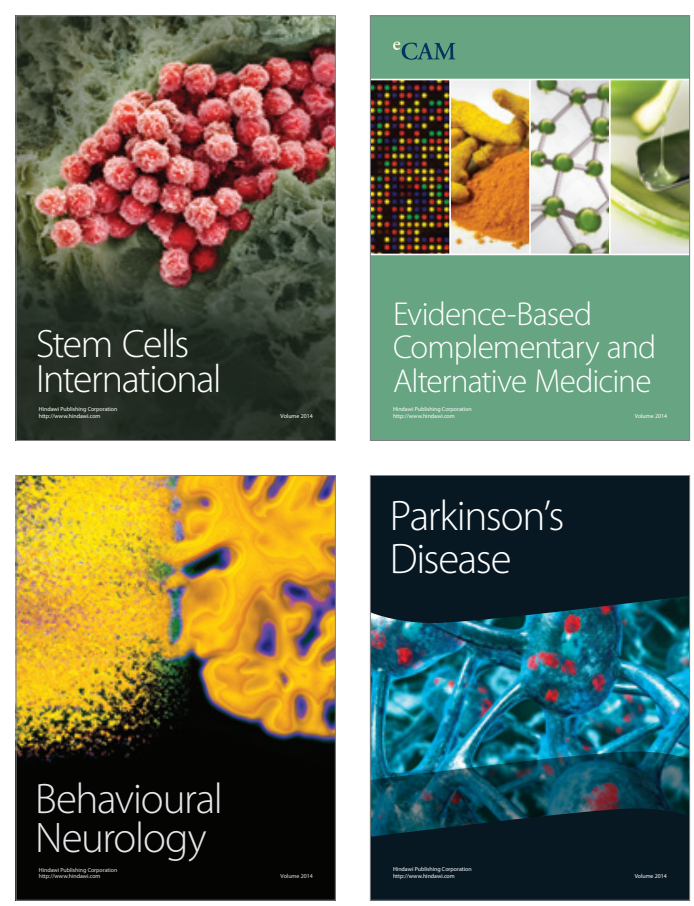

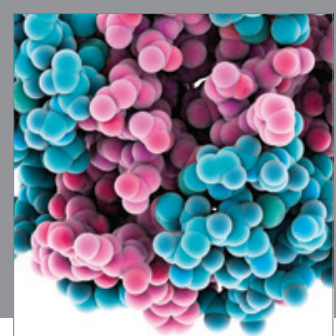

Journal of
Diabetes Research

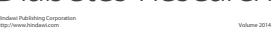

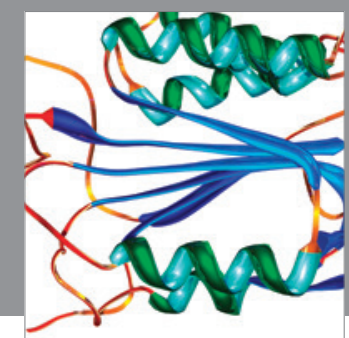

Disease Markers
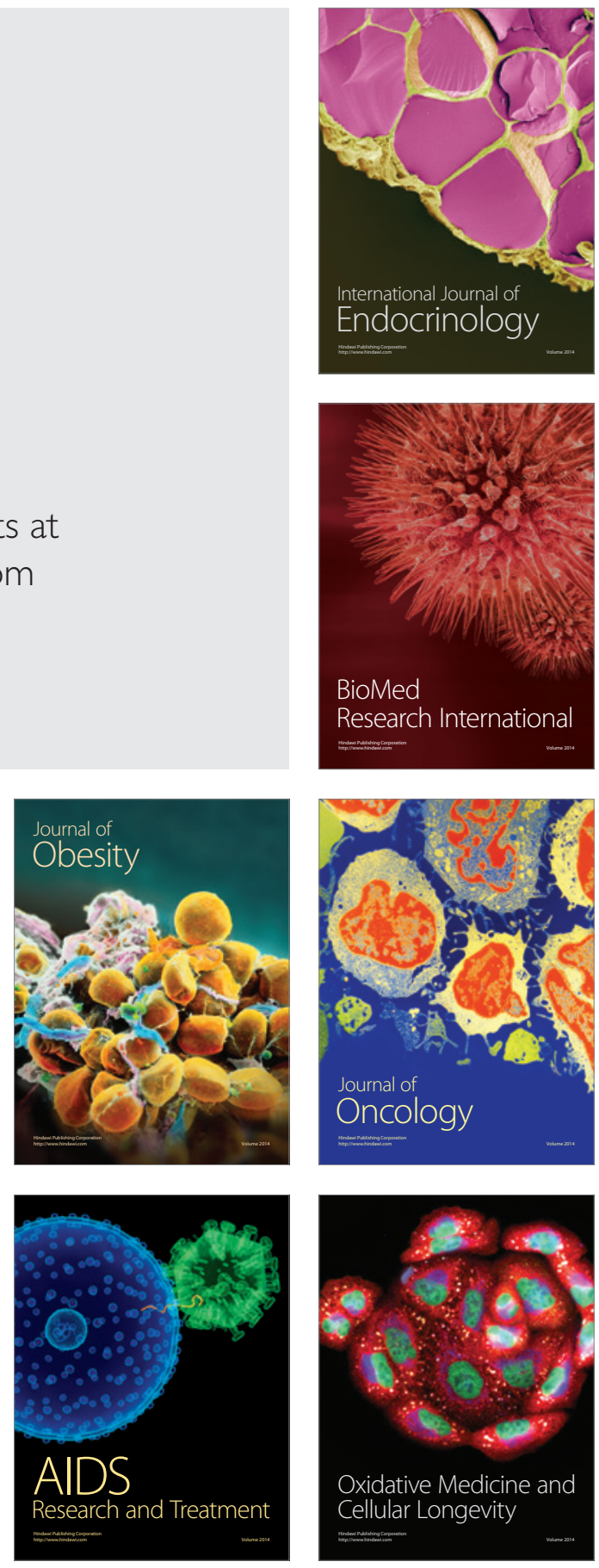